\title{
A comparative User Evaluation of Terminology Management Tools for Interpreters
}

\author{
Hernani Costa* Gloria Corpas Pastor Isabel Durán Muñoz \\ LEXYTRAD, University of Malaga, Spain \\ $\{$ hercos, gcorpas, iduran\} @uma.es
}

\begin{abstract}
When facing new fields, interpreters need to perform extensive searches for specialised knowledge and terminology. They require this information prior to an interpretation and have it accessible during the interpreting service. Fortunately, there are currently several terminology management tools capable of assisting interpreters before and during an interpretation service. Although these tools appear to be quite similar, they provide different kind of features and as a result they exhibit different degrees of usefulness. This paper aims at describing current terminology management tools with a view to establishing a set of features to assess the extent to which terminology tools meet the specific needs of the interpreters. Subsequently, a comparative analysis is performed to evaluate these tools based on the list of features previously identified.
\end{abstract}

\section{Introduction}

Professional interpreters frequently face different settings and specialised fields in their interpretation services and yet they always need to provide excellent results. They might be called to work for specialists that share a background knowledge that is totally or partially unknown to laypersons and/or outsiders (Will, 2007). When interpreters lack the necessary background knowledge or experience, they usually need to perform extensive searches for specialised knowledge and terminology in a very efficient way in order to supply this deficit and acquire the required information.

Even though there are several modes of interpretation, depending mainly on the timing/delay of the interpretation, the direction and the setting (cf. Pöchhacker, 2007), it is not possible for interpreters to collect the relevant specialised information during the interpretation service itself. Interpreters are required to find the necessary information prior to interpretation and have it accessible during the service, even though they sometimes are able to carry out searches during the service.

According to Rodríguez and Schnell (2009), terminology work is present in the whole process of preparation prior to an interpretation service. For example, interpreters become familiar with the subject field by searching for specialised documents, by extracting terms and looking for synonyms and hyperonyms, by finding and developing acronyms and abbreviations and by compiling a glossary. According to these authors, interpreters tend to compile in-house glossaries tailor to their individual needs as the main way to prepare the terminology of a given interpretation.

\section{Interpreters' Needs}

The potentialities of computers for improving interpreters' working conditions was realised a long time ago by Gile (1987). However, very little progress has been made so far. Costa, Corpas Pastor and DuránMuñoz (2014) offer a tentative catalogue of current language technologies for interpreters, divided into terminology tools for interpreters, note-taking applications for consecutive interpreting, applications for voice recording and training tools. This paper focus exclusively on terminology tools for interpreters with a view to performing a user evaluation.

As a rule, most interpreters seem to be unaware of the opportunities offered by language technologies. As far as terminology is concerned, interpreters continue to store information and terminology on scraps

\footnotetext{
${ }^{*}$ Hernani Costa is supported by the People Programme (Marie Curie Actions) of the European Union's Framework Programme (FP7/2007-2013) under REA grant agreement $\mathrm{N}^{\mathrm{o}} 317471$.

This work is licenced under a Creative Commons Attribution 4.0 International License.
} 
of paper or excel spreadsheets, while the use of technologies and terminology management tools is still very low. A study conducted by Moser-Mercer (1992:507, quoted in Bilgen, 2009) rejected the assumption that "interpreters' needs are identical to those of translators and terminologists" and intended to "survey how conference interpreters handle terminology documentation and document control and to offer some guidelines as to the interpretation-specific software tools for terminology and documentation management". The results of this study includes some key findings, such as the conclusion that most of the respondents were interested in exchanging terminological information and that they were open to using computers in their profession. According to these findings, Moser-Mercer (1992) highlighted that "software developers targeting the conference interpreting market must provide a tool that meets the specific needs of the interpreters and not just market translation tools" (ibid:511). More recent studies have also studied interpreters' current needs and practices regarding terminology management (Rodríguez and Schnell, 2009; Bilgen, 2009), and they also share the same findings: interpreters require specific tools to meet their needs, which are different from translators and terminologists. According to a survey conducted by Bilgen (2009), 85\% of respondents are open to using computers, yet conventional methods still prevail over the use of computerised methods of terminology management. The author observed that respondents had no or little experience with terminology management software, and those with some experience were most dissatisfied with the money and time they had to invest in them, and their overall experience was mediocre (ibid:66). Respondents indicated that their priorities were different from those identified in terminology literature in terms of terminological information stored, and the way in which term records are structured. This is an important aspect that differentiates the needs of interpreters and translators as regards definitions and contexts (Bilgen, 2009). Due to their working conditions, translators usually prefer to consult multiple definitions and contexts to find the best solution for the translation problem. On the contrary, interpreters will rarely have the time to go over multiple definitions, contexts, etc. to find the right one, and thus, they will need to store the most concise information to be able to consult it in the quickest and easiest way. Their responses in this survey also showed that the way they retrieve terminological information was context-specific, and that there was also a significant variation among individual interpreters. Flexibility is, therefore, of great importance to interpreters due to the variation of their context-specific terminology management practices, and on their individual preferences regarding the storage, organisation and retrieval of terminological information (ibid:92). Rodríguez and Schnell (2009), after a thorough analysis of interpreters' needs and in order to meet their requirements as regards terminology management tools, propose the possibility of developing small databases that vary according to the area of speciality or according to the conference and client. These mini-databases would be multilingual and include an option allowing the interpreter to switch the source and target languages. This assumption is in line with the Function Theory (Bergenholtz and Tarp, 2003; Tarp, 2008) and electronic multifunctional dictionaries (Spohr, 2009), which both defend the need to elaborate terminological entries according to the potential users. Rodríguez and Schnell (2009) recognise five features that would distinguish the interpreters' mini-databases from the terminology databases intended for translators: speed of consultation; intuitive navigation; possibility of updating the terminology record in the interpretation booth; considerable freedom to define the basic structure; and multiple ways of filtering data.

Accordingly, they also suggest the abandonment of the usual terminology methodology if the intention is to provide interpreters with specific glossaries tailored to their needs. The authors advance the use of a semasiological and associative methodology instead of the onomasiological approach, as "it does not adapt well to interpretation because the cognitive effort required by the onomasiological structures slows down the interpretation process" (ibid).

\section{Terminology Management Tools for Interpreters}

There are some specialised computer and mobile software that can be used to quickly compile, store, manage and search within glossaries. The most outstanding applications developed by/for interpreters are described in detail below. They can be typically used to prepare an interpretation, in consecutive interpreting or in a booth. These applications are quite similar to the look-up terminology tools currently 
used by translators (Durán Muñoz, 2012). In fact, some of them have been developed to cater for the needs of both translators and interpreters. Due to the lack of space, this article is focused on standalone applications, but other types of applications like Web-based (e.g. Interpreters' $\mathrm{Help}^{1}$ ) can also be used for the same purposes (Ruetten, 2014).

Intragloss $^{2}$ is a commercial Mac OS X software created specifically to help interpreters when preparing for an event by allowing them to manage glossaries. This application can be simply defined as a glossary and document management tool created to help the interpreter prepare, use and merge different glossaries with preparation documents, in more than 180 different languages. It allows to import and export glossaries from and to plain text, Microsoft Word and Excel formats. Every glossary imported to, or created in, is assigned to a domain glossary (considered the highest level of knowledge), which contains all the glossaries from the sub-areas of knowledge, named 'assignments'. The creation of an assignment glossary can be done in two different ways: either by extracting automatically all the terms from the domain glossary that appear in the imported documents, or by highlighting a term in the document, search for it on search sites (such as online glossaries, terminology databases, dictionaries and general Web pages) and manually add the new translated term to the assignment glossary. It is important to mention that the online search can be made within Intragloss. Another interesting feature is that Intraglosss permits to copy assignment glossaries and assignment entries from one assignment to another. The domain glossary may be multilingual as it can include several bilingual assignment glossaries. By a way of example, if we have two assignment glossaries English/French and Dutch/English, in the same domain, the domain glossary will be French/English/Dutch, i.e. multilingual. Finally, Intragloss also allows to manually add meta-information to each glossary entry (see Fig. 1a).

In short, Intragloss is an intuitive and easy-to-use tool that facilitates the interpreters' terminology management process by producing glossaries (imported or created ad hoc), by searching on several websites simultaneously and by highlighting all the terms in the documents that appear in the domain glossary. However, it is currently platform dependent and only works on Mac OS X platforms.

InterpretBank $^{3}$ is a simple terminology and knowledge management software tool designed both for interpreters and translators using Windows and Android. It helps to manage, learn and look up glossaries and term-related information. Due to its modular architecture (see Fig. 1b), it can be used to guide the interpreter during the entire workflow process, starting from the creation and management of multilingual glossaries (TermMode), passing through the study of these glossaries (MemoryMode), and finally allowing the interpreter to look up terms while in a booth (ConferenceMode). InterpretBank also has an Android version called InterpretBank Lite. This application is specifically designed to access bi- or trilingual glossaries previously created with the desktop version. It is useful when working as a consecutive, community or liaison interpreter, when a quick look up at the terminology list is necessary.

InterpretBank has a user-friendly, intuitive and easy-to-use interface. It allows us to import and export glossaries in different formats (Microsoft Word, Microsoft Excel, simple text files, Android and TMEX) and suggests translation candidates by taking advantage of online translation portal services, such as Wikipedia, MyMemory and Bing. However, it is platform-dependent (it only works on Windows and Android), does not handle documents, only glossaries and requires a commercial license.

Interplex $\mathbf{U E}^{4}$ is a user-friendly multilingual glossary management programme that can be used easily and quickly in a booth while the interpreter is working. Instead of keeping isolated word lists, it allows to group all terms relating to a particular subject or field into multilingual glossaries that can be searched in an instant. As we can see in Fig. 1c, this programme permits to have several glossaries open at the same time, which is a very useful feature if the working domain is covered by more than one glossary. Similar to the previous analysed programmes, Interplex UE also allows to import and export glossaries from and to Microsoft Word, Excel, and simple text files. Interplex UE runs on Windows; nevertheless, it has a simpler version for iOS devices, one named Interplex Lite, for iPhone and iPod Touch, and another named Interplex HD, for iPad. Both glossaries and multi-glossary searchers offer the functionality of

\footnotetext{
${ }^{1}$ www. interpretershelp.com

2 intragloss.com

${ }^{3}$ www . interpretbank . de

4 wWw. fourwillows. com
} 
viewing expressions in each of the defined languages.

In general, Interplex UE has a user-friendly interface and it is regularly updated. It allows to import and export glossaries from and to Microsoft Word and Excel formats. However, it, too, is platform dependent (only works on Windows and iOS), does not handle documents, only glossaries, and requires a commercial license.

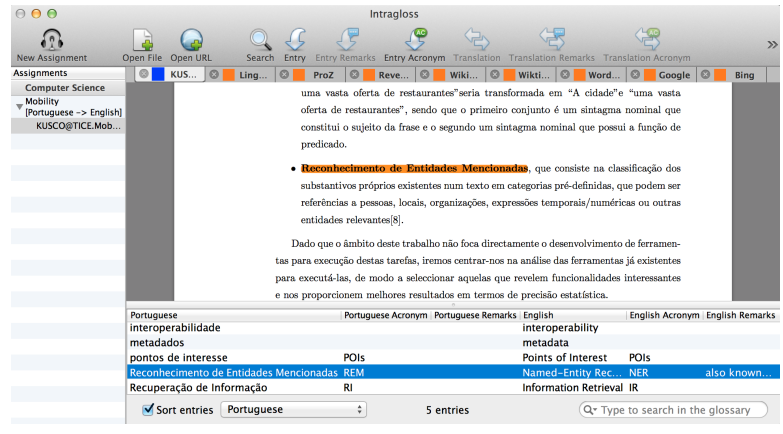

(a) Intragloss.

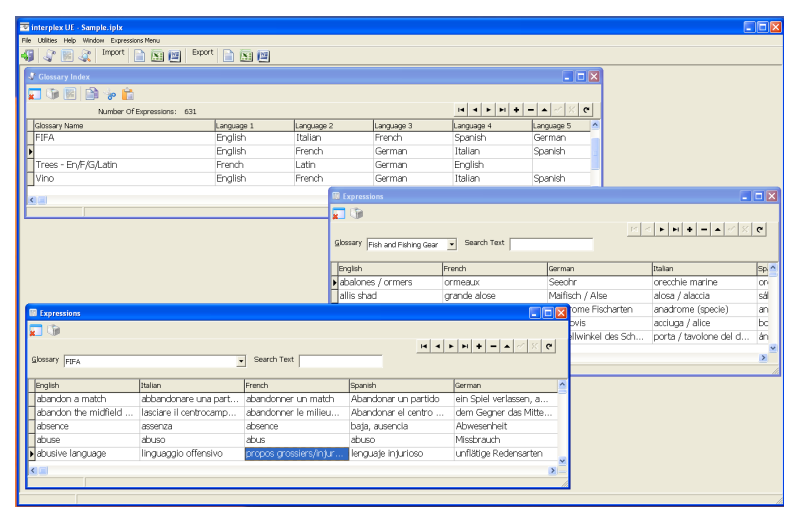

(c) Intraplex.

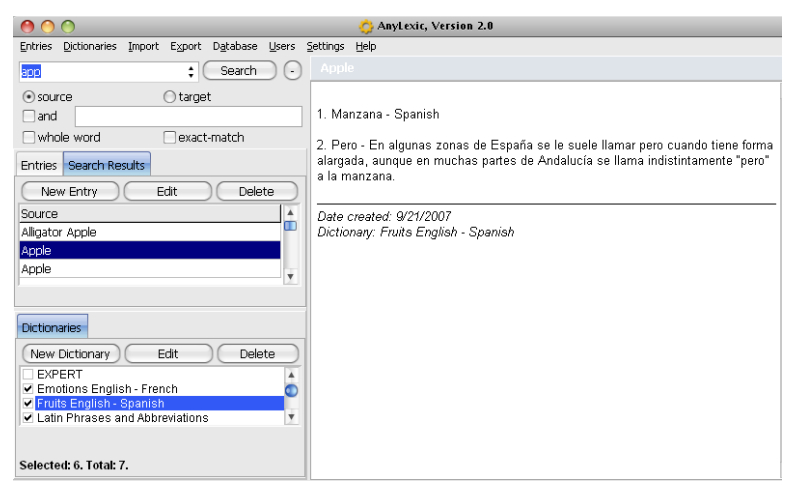

(e) AnyLexic.

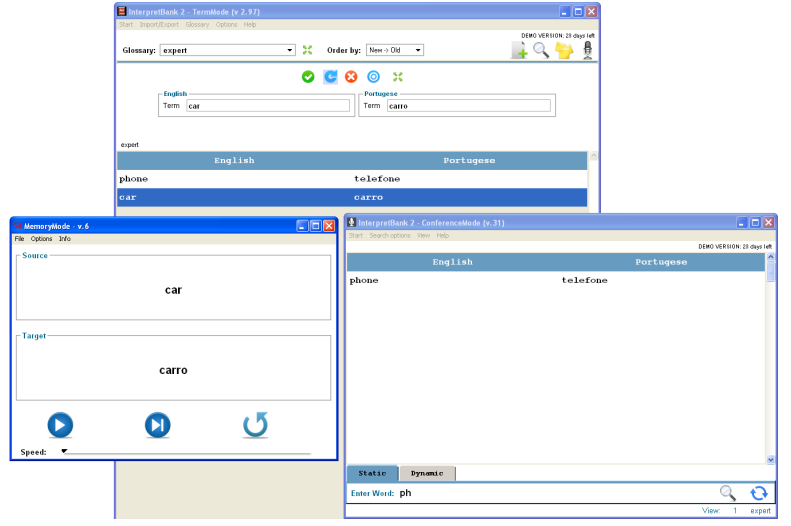

(b) InterpretBank.

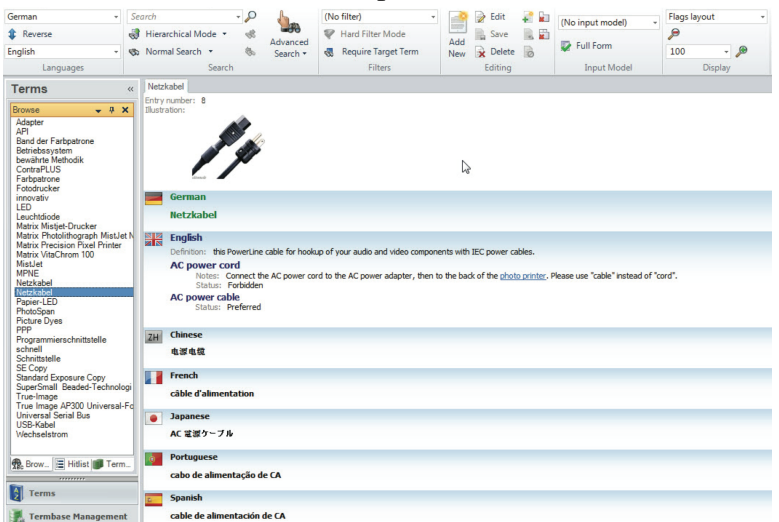

(d) SDL MultiTerm.

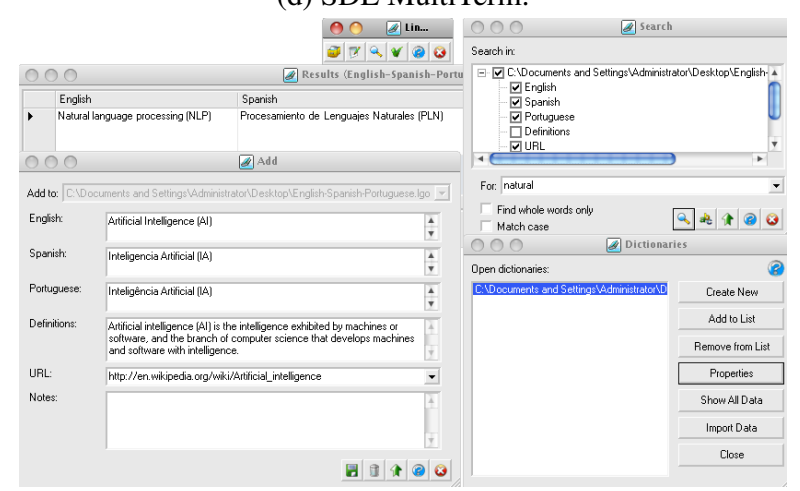

(f) Lingo.

Figure 1: Screenshots of various terminology managment tools.

SDL MultiTerm Desktop ${ }^{5}$ is a commercial terminology management tool developed for Windows that provides one solution to store and manage multilingual terminology. MultiTerm was first launched in 1990 by Trados GmbH but in 2005 the company was acquired by $\mathrm{SDL}^{6}$, which renamed MultiTerm to SDL MultiTerm. Today, SDL MultiTerm is a terminology management tool commercialised by SDL as a standalone application, which has been improved according to the translators' needs. Alternatively, MultiTerm can be used within the SDL Trados Studio ${ }^{7}$ as an integrated tool. As translators can

\footnotetext{
${ }^{5}$ WWW.sdl.com/products/sdl-multiterm/desktop.html

${ }^{6}$ WWW . sdl . com

${ }^{7}$ WWW.sdl.com/products/sdl-trados-studio
} 
easily edit and add terminology within SDL Trados Studio, MultiTerm helps to improve the efficiency of the translation process and promotes high-quality translated content with real-time verification of multilingual terminology. This application is very complete because it allows to store an unlimited number of terms in a vast number of languages; imports and exports glossaries from and to different technology environments, such as Microsoft Excel, XML, TBX and several other proprietary formats; permits to manually add a variety of meta-data information, such as synonyms, context, definitions, associated project, part-of-speech tags, URLs, etc. Apart from the previous mentioned descriptive fields, MultiTerm also allows the user to insert illustrations to the terms in the terminology database (which can be stored either locally or, for collaborative purposes, in a remote server). It is important to mention that this visual reference feature is very useful specially to interpreters and translators dealing with unfamiliar terms. Moreover, MultiTerm has an advanced search feature that permits to search not only the indexed terms but also in their descriptive fields, or create filters to make custom searches within specific fields, like language, definition, part-of-speech, etc. Nevertheless, the most interesting feature about MultiTerm is its concept-oriented feature, i.e. each entry in MultiTerm corresponds to a single concept, which can be described by different terms in both source and target language. This detail is very important because it allows the user to centralise and customise the terms with more information, such as different possible translations and their corresponding contexts (see Fig. 1d).

In general, MultiTerm can be seen as an advanced multilingual terminology tool with an intuitive and easy-to-use interface. Although MultiTerm was originally designed for translators, it can also be used by interpreters. Its main advantage to interpreters, when compared with other terminology tools, is twofold: it allows to add several translation terms in one entry and permits to customise a wide variety of descriptive fields, such as illustrations, associated projects, definitions, etc. However, it can only be used on Windows, does not handle documents and there is no demo version available.

AnyLexic $^{8}$ is an easy-to-use terminology management tool developed for Windows with a simple and intuitive interface. It was not designed to tie any particular terminological requirement, instead it aims to help the interpreter prepare, use and manage different glossaries or dictionaries. AnyLexic can be described as a robust terminology management tool, as it enables users to easily create and manage multiple mono-, bi- or multilingual glossaries in any language and to import and export glossaries from and to Microsoft Excel, plain text and AnyLexic Exchange Format (AEF). In addition, each entry in the glossary can have multiple translation equivalents in the target language along with notes. The search for records in the database allow users to combine different options, such as search for all source terms or translation candidates and associated notes. In addition, the search can be performed within one or multiple glossaries (see Fig. 1e). Another interesting feature in AnyLexic is the way that records can be displayed using different templates with configurable text colour, background colour, font size and text format. Besides, it is possible to create our own template for displaying the records. With the purpose of simplifying the teamwork process, this tool has an additional option to exchange any glossary with other AnyLexic users by either using the AEF proprietary format or by accessing a remote glossary, a very useful feature when co-operating with other interpreters or translators on a project.

In general, AnyLexic is an easy and convenient terminology database managing software for working with terminology, creating, editing and exchanging glossaries when working under one project both alone or with other working partners. However, it only works on Windows platforms and even though an evaluation version is available for 30 days, it requires a commercial license.

Lingo ${ }^{9}$ is a commercial Windows terminology management tool designed to create and manage terminology databases, whether mono- or multilingual. It can import from and export to TMX and plain text. Its main features are: creation and management of any number of specialised glossaries/dictionaries in any language; can handle large files (i.e. over 50K entries); it allows users to have several glossaries open at the same time; and it has a rapid and easily configurable search functionality that can be customised to search for all terms, translation candidates and associated descriptive fields, either in all glossaries or in a specific one. Another interesting feature is the drag and drop functionality, which

\footnotetext{
${ }^{8}$ wWw. anylexic. com

${ }^{9}$ wWw. lexicool.com/soft_lingo2.asp
} 
allows to easily insert words into Microsoft documents, for instance.

As we can see in Fig. 1f, Lingo is a simple and user-friendly software that offers an effective way to create and manage multilingual glossaries in any language. Additionally, it permits to manually add an infinite number of customised fields into each entry, such as definitions, URLs, synonyms, antonyms, contextual information, notes or any other desirable field. However, it is platform dependent and does not import from or export to common formats like Microsoft Word or Excel.

UniLex $^{10}$ is a free terminology management tool created by Acolada GmbH for Windows. It aims to help interpreters and translators prepare, use and manage bilingual glossaries or dictionaries in approximately 30 different languages. UniLex offers a variety of search functions and the possibility to combine user glossaries or dictionaries with a full range of dictionaries available in the UniLex series (e.g. Blaha: Pocket Dictionary of Automobile Technology German/English), which can be acquired as single user versions or as network versions for collaborative purposes. UniLex can also be used in a network environment, which allows users to exchange glossaries or dictionaries. Nevertheless, this additional feature requires a commercial license.

In general, UniLex is not only capable of managing user bilingual glossaries or dictionaries, but also dictionary titles from renowned publishers, which are sold by the company to be consulted within UniLex. However, it only works on Windows and does not handle multilingual glossaries.

The Interpreter's Wizard ${ }^{11}$ is a free iPad application capable of managing bilingual glossaries in a booth. It is a simple, fast and easy-to-use application that helps the interpreter to search and visualise terminology in seconds. The system includes rapid and easily configurable search functionality that can be customised to search for all terms, translation candidates either in all glossaries or in a specific one. Nevertheless, all the imported glossaries need to be previously created and converted online to the proprietary format, and it does not allow users to export glossaries.

\subsection{Comparative Analysis}

Despite the aforementioned terminology tools can be used to prepare a given interpretation according to the interpreters' requirements identified in section 2, these systems differ from one another in their functionalities, practical issues and degrees of user-friendliness. Therefore, it is necessary to establish a set of specific and measurable features that permit us to assess and distinguish the different tools concerning users' needs in such a way that the results would be useful for both potential users as well as to the designers of such systems. Departing from the conclusions drawn from the literature review (see section 2) and the description of the terminology tools analysed in section 3, we provide in this section an analysis of these tools based on our own practical set of measurable features. For instance, the "freedom to define the basic structure" identified by Rodríguez and Schnell (2009) was reformulated into several practical measurable features, such as " $\mathrm{N}^{\mathrm{O}}$ of descriptive fields", " $\mathrm{N}^{\mathrm{o}}$ of working languages" and " $\mathrm{N}^{0}$ of languages per glossary". Moreover, the possibility of "developing multilingual mini-databases", also identified in their study, was reconsidered as measurable features by means of the following criteria: "Manages multiple glossaries" and " $\mathrm{N}^{0}$ of languages per glossary". Another example is the "Remote Glossary Exchange" measurable feature, which was inferred from the study conducted by Bilgen (2009), who identified the need to exchange terminological information.

After a careful analysis of the priorities for the design and features to be included in a terminology management tool for interpreters, 15 features were identified, 5 of which were classified as fundamental to a terminology tool (10 points) and 10 as secondary (5 points). Then, these features were used to evaluate the eight tools presented in section 3 and to investigate which one is the most complete. The first considered feature clarifies if the tools were designed to handle multiple glossaries in their interfaces at same time (Manages multiple glossaries). The next two features are somehow related. The $\mathbf{N}^{0}$ of possible working languages describes how many different working languages are permitted by the application. Then, considering these working languages, how many of them can be used at the same time per glossary ( $\mathbf{N}^{\mathbf{0}}$ of languages per glossary allowed). The next feature is related with

\footnotetext{
${ }^{10}$ www . acolada.de/unilex.htm

${ }^{11}$ the-interpreters-wizard.topapp.net
} 


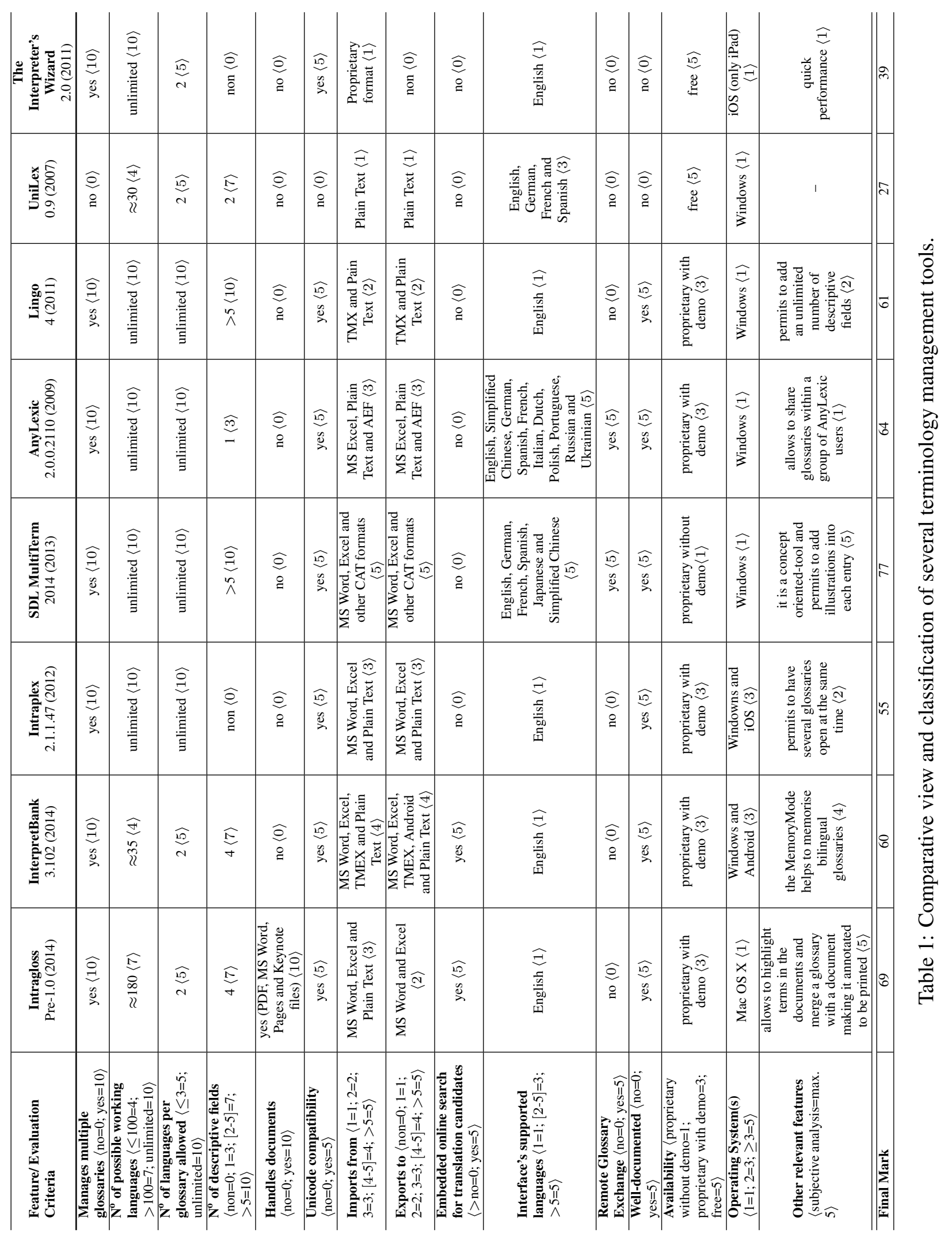


all types of descriptive fields that these tools allow to add to each glossary entry ( $\mathbf{N}^{\mathbf{0}}$ of descriptive fields). The possibility of managing terminology with preparation documents (Handles documents) is another relevant feature for interpreters seeking for tools capable of highlighting terms in documents, for example. Equally import is the Unicode support (Unicode compatibility) as it provides a unique number for every character, no matter what the platform, the program, or the language is. In other words, an application that supports full Unicode means that it has support for any ASCII or non-ASCII language, such as Hebrew or Russian, two non-ASCII languages. Imports from and Exports to, as its name suggests, represents the supported input and output formats. The Embedded online search for translation candidates is a relevant add-in for terminology tools, as it permits to focus the search for terminological candidates within the tool. Despite the fact that all the tools have English as a default language, the support of multiple languages (Interface's supported languages) is another important feature as it allows to increase the number of potential users that a terminology tool can reach. The Remote glossary exchange feature is important when co-operating with other working partners remotely is required. The next three features are related with the available documentation, their availability and platform dependency (Well-documented, Availability and Operating System(s), respectively). Finally, the last row presents some unique characteristics along with some relevant comments (Other relevant features).

Based on this comparative analysis, none of the investigated terminology tools exhibit all the proposed features. Nevertheless, SDL MultiTerm and Intragloss are the best classified with 77 and 69 points out of 100, respectively. This is not surprising because SDL MultiTerm is the most expensive tool nowadays available on the market and, apart from that, it has been developed for more than 20 years. Its flexibility to easily store, manage and search for multilingual terminology and definitions is just an example of the features that meet the specific needs of an interpreter. The score of Intragloss, released last year as a beta version, is neither surprising due to its novelty and design purposes, i.e. it was developed by interpreters for interpreters and thus corresponds better to their needs. On the other hand, UniLex and The Interpreter's Wizard tools got the worst scores due to the lack of features offered. About the remaining tools (AnyLexic, Lingo, InterpretBank and Intraplex) we can say that they have similar features, which resulted in similar scores $(64,61,60$ and 55 , respectively).

\section{Conclusion}

This paper presents an overview of the most relevant features that terminology management tools should have in order to help interpreters before and during the interpretation process. Eight terminology tools are discussed and a comparative analysis is performed to evaluate them on the bases of the set of features previously identified. This comparative analysis not only aims at highlighting some of the features that interpreters can expect from the currently available terminology management tools on the market, but also intended to help them choose a specific tool for a given interpretation project. Table 1 provides interpreters with a comprehensive and up-to-date review of the currently available terminology tools on the market. It is envisaged to serve as a concise guide to help interpreters choose the terminology management tool that best caters for their specific needs, in order to help them work more efficiently, store and share terminology more easily, as well as save time when a looking for a specific feature most suited to a specific interpreting service.

Although most of the analysed tools could be considered to be very flexible when searching for terminology within glossaries, it appears that none of them fulfil all needs of interpreters. It is worth mentioning that some tools require a steep learning curve while others imply a significant financial investment (e.g. Lingo and SDL MultiTerm, respectively). Moreover, some tools are fairly basic and more orientated towards creating and managing bilingual or multilingual glossaries rather than more comprehensive terminology records with supporting information (e.g. UniLex and The Interpreter's Wizard).

Given that quality terminology management ranks high in their priorities, it would seem that there is a pressing need to design terminology management tools tailored to assist interpreters in the preparation stage, before their interpreting service or during it. In this respect, it would be necessary to identify the exact needs of interpreters (which are likely to be different from translators). 


\section{Acknowledgements}

The research reported in this article has been partially carried out in the framework of the Educational Innovation Project TRADICOR (PIE 13-054, 2014-2015); the R\&D project INTELITERM $\left(\mathrm{n}^{\mathrm{o}}\right.$ FFI201238881, 2012-2015), and the R\&D Project for Excelence TERMITUR (ref. $\mathrm{n}^{\circ}$ HUM2754, 2014-2017).

\section{References}

Henning Bergenholtz and Sven Tarp. 2003. Two opposing theories: On H.E. Wiegand's recent discovery of lexicographic functions. Hermes, Journal of Linguistics, 31:171-196.

Baris Bilgen. 2009. Investigating Terminology Management for Conference Interpreters. MA dissertation, University of Otawa, Otawa, Canada.

Hernani Costa, Gloria Corpas Pastor, and Isabel Durán Muñoz. 2014. Technology-assisted Interpreting. MultiLingual 143, 25(3):27-32, April/May.

Isabel Durán Muñoz. 2012. Meeting Translators' Needs: Translation-oriented Terminological Management and Applications. The Journal of Specialised Translation, 18:77-92. Available at: http://www . jostrans . org/issue18/art_duran.pdf (Accessed 30 June 2014).

Daniel Gile. 1987. La terminotique en interprétation de conférence: un potentiel à exploiter. Meta: Translators' Journal, 32(2):164-169, June.

Barbara Moser-Mercer. 1992. Banking on Terminology: Conference Interpreters in the Electronic Age. Meta: Translators' Journal, 37(3):507-522, September.

Franz Pöchhacker. 2007. Introducing Interpreting Studies. London and New York: Routledge, $2^{\text {nd }}$ edition.

Nadia Rodríguez and Bettina Schnell. 2009. A Look at Terminology Adapted to the Requirements of Interpretation. Language Update, 6(1):21-27. Available at: http://www.btb.termiumplus . gc.ca/tpv2guides/guides/favart/index-eng.html?lang=eng\&lettr=indx_ autr8gijKBACeGnI\&page=90HAHvmF zkgE . html (Accessed 30 June 2014).

Anja Ruetten. 2014, June 30. Booth-friendly terminology management revisited - 2 newcomers. Retrieved from: http://blog. sprachmanagement. net/ ?p=305 (Accessed 30 June 2014).

Dennis Spohr. 2009. Towards a Multifunctional Electronic Dictionary Using a Metamodel of User Needs. In eLexicography in the $21^{\text {st }}$ century: New challenges, new applications, Louvain-La-Neuve, Belgium. Presses Universitaires de Louvain.

Sven Tarp. 2008. Lexicography in the Borderland Between Knowledge and Non-knowledge: General Lexicographical Theory with Particular Focus on Learner's Lexicography. Lexicographica: Series maior. Walter de Gruyter, $1^{\text {st }}$ edition.

Martin Will. 2007. Terminology Work for Simultaneous Interpreters in LSP Conferences: Model and Method. In Heidrun Gerzymisch-Arbogast and Gerhard Budin, editors, Proc. of the Marie Curie Euroconferences MuTra: LSP Translation Scenario, EU-High-Level Scientific Conference Series, Vienna, Austria. 